\title{
Exogenous surfactant attenuation of ischemia-reperfusion injury in the lung through alteration of inflammatory and apoptotic factors
}

\author{
Bart P. van Putte, PhD, MD, ${ }^{\mathrm{a}, \mathrm{c}}$ Pieter M. Cobelens, PhD, ${ }^{\mathrm{a}, \mathrm{b}}$ Niels van der Kaaij, PhD, ${ }^{\mathrm{d}}$ Burkhard Lachmann, PhD, MD, \\ Annemieke Kavelaars, $\mathrm{PhD},{ }^{\mathrm{b}}$ Cobi J. Heijnen, $\mathrm{PhD},{ }^{\mathrm{b}}$ and Jozef Kesecioglu, $\mathrm{PhD}, \mathrm{MD}^{\mathrm{a}}$
}

\begin{abstract}
Objective: Lung ischemia-reperfusion injury is associated with impaired gas exchange from increased edema formation and surfactant inactivation. Surfactant replacement therapy is believed to improve gas exchange and lung function, but its effect on inflammation is less well understood. We therefore examined the effects of exogenous surfactant on inflammatory and apoptotic factors in the lung in a rat model of lung ischemia-reperfusion injury.
\end{abstract}

\begin{abstract}
Methods: The left lung in rats was subjected to ischemia for 120 minutes and reperfusion for as long as 240 minutes. Sham-treated animals underwent sham surgery and mechanical ventilation for equivalent times. Rats received porcine surfactant or saline solution intratracheally either before or just after ischemia. Lungs were analyzed histopathologically and for expressions of inducible nitric oxide, cytokines, and caspase-3.

Results: Lung ischemia-reperfusion injury resulted in worse lung histopathologic characteristics than in shamoperation animals. At 2 hours of reperfusion, lung ischemia-reperfusion injury animals showed increased pulmonary caspase-3 expression. Moreover, lung ischemia-reperfusion injury resulted in inducible nitric oxide expression at all time points. Exogenous surfactant resulted in less inflammatory cell infiltration and edema in the lungs relative to saline-treated animals. Surfactant decreased activated caspase-3 expression and increased inducible nitric oxide expression relative to saline-treated animals. At 4 hours of reperfusion, surfactant increased interleukin 6 and 10 expressions in the lung.
\end{abstract}

Conclusion: This study showed a significant improvement in lung histologic characteristics after surfactant therapy, accompanied by reduced apoptosis and increased anti-inflammatory cytokine levels. Interestingly, surfactant therapy also increased pulmonary inducible nitric oxide expression.

Lung ischemia-reperfusion injury (LIRI) is a significant cause of early morbidity and mortality after lung transplantation. LIRI is characterized by acute hypoxic respiratory failure resulting from noncardiogenic pulmonary edema caused by an increased permeability of the alveolar capillary barrier. Several data from experimental studies for acute lung injury suggest that exogenous surfactant can improve lung function and outcome by decreasing edema formation, reducing protein leakage, and improving histopathologic state. $^{1-6}$ Interestingly, surfactant also has shown strong immunomodulatory properties in vitro. For instance, surfactant proteins $\mathrm{A}$ and $\mathrm{D}$ are able to regulate toll-like receptors, resulting in altered production of inflammatory mediators. ${ }^{7}$ Furthermore, surfactant preparations containing surfactants

\footnotetext{
From the Department of Intensive Care Medicine ${ }^{\mathrm{a}}$ and the Laboratory for Psychoneuroimmunology, ${ }^{\mathrm{b}}$ University Medical Center, Utrecht, The Netherlands, the Department of Cardiothoracic Surgery, St Antonius Hospital, Nieuwegein, The Netherlands, ${ }^{c}$ and the Department of Anaesthesiology, Erasmus Medical Center, Rotterdam, The Netherlands. ${ }^{\mathrm{d}}$

Received for publication Feb 18, 2008; revisions received July 21, 2008; accepted for publication Aug 28, 2008.

Address for reprints: Bart P. van Putte, PhD, MD, Department of Cardiothoracic Surgery, St. Antonius Hospital, Koekoekslaan 1, Nieuwegein, The Netherlands (E-mail: bvanputte@yahoo.com).

J Thorac Cardiovasc Surg 2009;137:824-8

$0022-5223 / \$ 36.00$

Copyright (c) 2009 by The American Association for Thoracic Surgery

doi: $10.1016 /$ j.jtcvs. 2008.08 .046
}

$\mathrm{B}$ and $\mathrm{C}$ reduce proinflammatory cytokine production by stimulated human alveolar macrophages and peripheral blood monocytes in vitro. ${ }^{8-10}$ Despite the clear effects of surfactant in vitro, data on the immunomodulatory effects of surfactant in vivo are scarce and contradictory. For instance, Vreugdenhil and colleagues ${ }^{11}$ showed exogenous surfactant to restore lung function, but with no effect on proinflammatory cytokine expression in the lung. In line with these data, several groups showed in different models that exogenous surfactant had no effect on proinflammatory mediators. ${ }^{12-14}$ On the other hand, Stamme and coworkers ${ }^{15}$ reported increased tumor necrosis factor $\alpha$ and interleukin (IL) 6 production in ventilation-induced lung injury, whereas Rasaiah and colleagues ${ }^{16}$ reported decreased tumor necrosis factor $\alpha$ and IL-6 production in sepsis-induced lung injury. Our aim in this study was to investigate the immunomodulatory effects of surfactant on inflammatory mediators and apoptosis in a rat model of LIRI.

\section{MATERIALS AND METHODS \\ Animals \\ Male Sprague-Dawley rats, obtained from Harlan (Harlan Netherlands BV, Horst, The Netherlands), were used. Animals were treated in accor- dance with the Animal Welfare Act and in compliance with the Guide for the Care and Use of Laboratory Animals (www.nap.edu/catalog/5140. html). All experiments were approved by the ethical committee of the Erasmus Medical Center (Rotterdam, The Netherlands).}




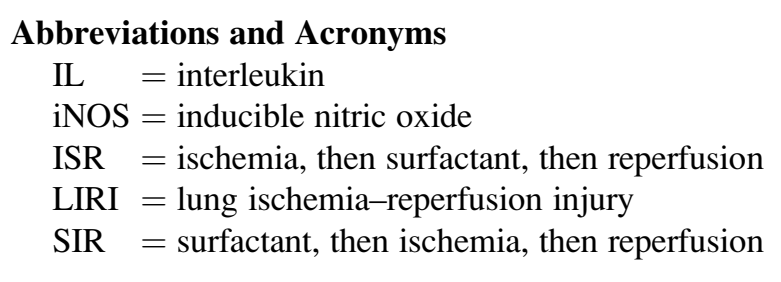

\section{Study Design}

Eighty-four rats were anesthetized with a gas mixture $(65 \%$ nitrous oxide, $33 \%$ oxygen, and $2 \%$ isoflurane) and subsequently intubated and mechanically ventilated $\left(12 \mathrm{~cm} \mathrm{H}_{2} \mathrm{O}\right.$ peak inspiratory pressure, $4 \mathrm{~cm} \mathrm{H} \mathrm{H}_{2} \mathrm{O}$ positive end-expiratory pressure, $50 \%$ inspired oxygen fraction, a frequency of 40 breaths/minute, and a ratio of inspiration to expiration time of 1:2). Anesthesia was continued with pentobarbital $(60 \mathrm{mg} / \mathrm{mL})$, and muscle relaxation was achieved with pancuronium bromide $(2 \mathrm{mg} / \mathrm{mL})$, both repeated every hour.

Except for the sham-operated group, which underwent all operative interventions but without induction of ischemia, all rats underwent 2 hours of warm ischemia of the left lung caused by clamping of the pulmonary hilum, followed by 30,120 , or 240 minutes of reperfusion (all groups $n=7$ ). Rats were treated by intratracheal instillation of surfactant (natural porcine surfactant, HL-10, $200 \mathrm{mg} / \mathrm{kg}$; LEO Pharma A/S, Ballerup, Denmark) either before (surfactant, then ischemia, then reperfusion [SIR]) or just after (ischemia, then surfactant, then reperfusion [ISR]) ischemia. Control rats received saline solution intratracheally before start of ischemia. At the end of reperfusion, the rats were killed with an overdose of pentobarbital.

\section{Surfactant and Surfactant Administration}

Surfactant was dissolved in saline solution $(45 \mathrm{mg} / \mathrm{mL})$ and administered intratracheally at a dose of $200 \mathrm{mg} / \mathrm{kg}$, resulting in surfactant exposure of both lungs.

All SIR rats received surfactant intratracheally after they were briefly anesthetized and intubated. Three dosages (each instillation included a third of the total volume per rat) were administered to each rat during 1 hour before the start of ischemia. After each surfactant instillation, the animals recovered from anesthesia while breathing spontaneously, resulting in homogeneous distribution in both lungs. The control rats received saline solution in the same volume and according to the same protocol as the SIR rats. The ISR animals had surfactant instillation after 2 hours of ischemia. Immediately after release of the clamp at the start of reperfusion, the whole dose of $200 \mathrm{mg} / \mathrm{kg}$ was administered intratracheally in a single instillation. After recruitment, pressure-controlled ventilation was temporarily augmented to achieve homogeneous distribution of surfactant.

\section{Western Blotting}

Total lung homogenates were prepared with tissue lysis buffer and a protease inhibitor cocktail (Sigma-Aldrich Co, St Louis, Mo). From 10 to $30 \mu \mathrm{g}$ protein was separated by either $10 \%$ or $15 \%$ sodium dodecyl sulfate polyacrylamide gel electrophoresis and analyzed for inducible nitric oxide (iNOS) expression (Santa Cruz Biotechnology, Inc, Santa Cruz, Calif) and cleaved caspase-3 expression (Santa Cruz), respectively, by immunoblot analysis. $\beta$-Actin (Santa Cruz) was used as loading control. Immunoreactivity was detected by enhanced chemiluminescence (ECL; Amersham plc, Little Chalfont, UK).

\section{Cytokines}

IL-6 (BD Biosciences, San Jose, Calif) and IL-10 (BD Biosciences) cytokine levels were measured in total lung homogenates by enzyme-linked immunosorbent assay according to manufacturer protocol.

\section{Lung Histologic Study}

Lung tissue specimens were fixed in formalin, dehydrated, cleared, and embedded in paraffin. Specimens were cut into $8-\mu \mathrm{m}$ serial sections and stained with hematoxylin and eosin.

\section{Statistical Analysis}

All data are presented as mean $\pm \mathrm{SE}$. All parameters were analyzed by 1-way analysis of variance, followed by Bonferroni post hoc test.

\section{RESULTS \\ Histologic Study}

Lung specimens of the control animals were characterized by severe pathologic changes at all reperfusion time points. At 30 minutes of reperfusion, atelectasis and thickened alveolar septa that had been infiltrated by inflammatory cells were observed (Figure 1). Alveolar edema and bleeding appeared to be more prominent at 240 minutes of reperfusion.

Interestingly, both surfactant groups (SIR and ISR) only showed slightly thickened alveolar septa, slight alveolar edema, and minimal infiltration of inflammatory cells at all reperfusion time points relative to saline-treated animals (Figure 1).

\section{Inflammatory Mediators}

Western blot analysis revealed iNOS expression in the control group at all reperfusion time points, whereas iNOS was undetectable in the sham-operated group $(P<.001$; Figure 2). Surfactant treatment decreased iNOS expression at 30 minutes of reperfusion in both the SIR and ISR groups $(P<.001)$. Interestingly, iNOS expression was significantly increased at 120 minutes of reperfusion in the SIR group relative to the control group $(P<.001)$, and a delayed response was observed in the ISR group, resulting in higher levels of iNOS at 240 minutes of reperfusion $(P<.01$; Figure 2$)$.

IL-6 expression in the control group remained stable at all reperfusion time points, being significantly higher at 240 minutes of reperfusion relative to the sham-operated group $(P<.05$; Figure 3). Surfactant treatment increased IL-6 expression relative to control at all time points only in the control group $(P<.05)$. In the ISR group, IL-6 was only elevated relative to control at 240 minutes of reperfusion $(P<.01$; Figure 3$)$.

IL-10 expression in the control group dropped significantly as a function of time, being significantly lower at 240 minutes of reperfusion relative to the sham-operated group $(P<.05$; Figure 4$)$. After surfactant treatment, significantly higher IL-10 expressions were observed at $240 \mathrm{~min}$ utes of reperfusion in both the SIR and ISR groups $(P<.01$ SIR, $P<.001$ ISR; Figure 4).

\section{Apoptosis}

Control animals showed increased levels of the proapoptotic marker cleaved caspase-3 relative to the sham-operated group only at 120 minutes of reperfusion $(P<.001)$. At 30 and 240 minutes of reperfusion, caspase-3 levels were similar 


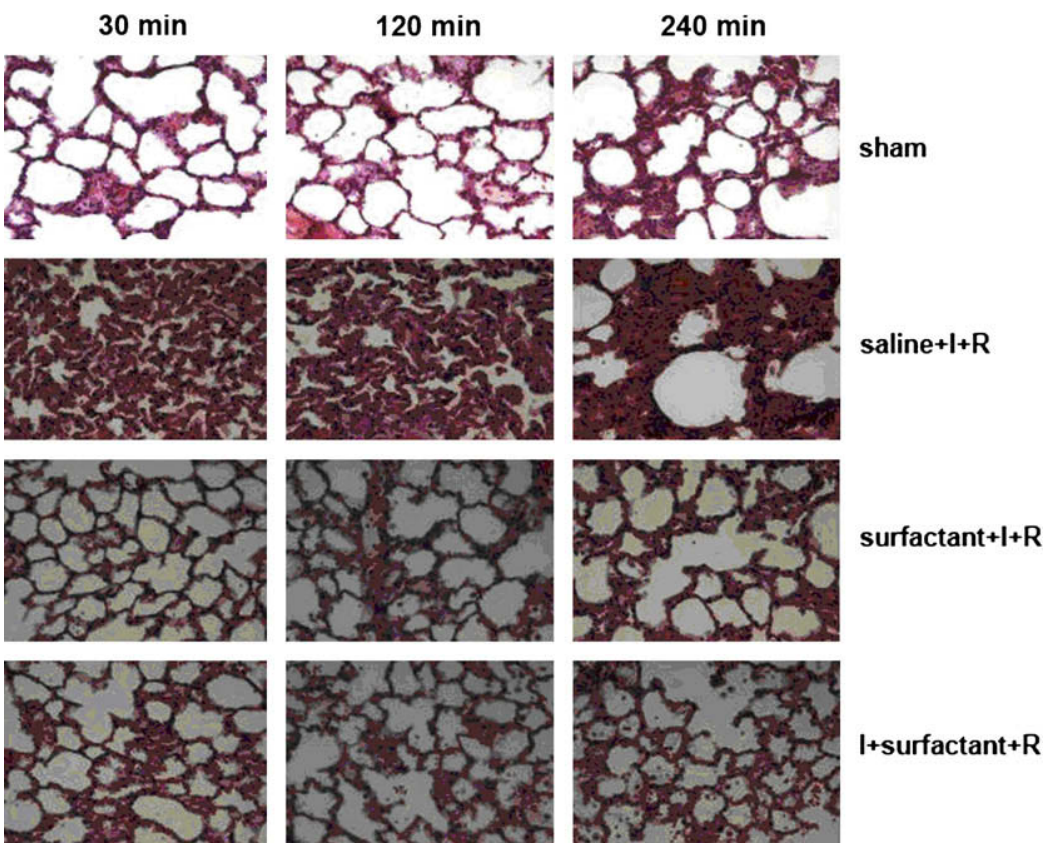

FIGURE 1. Representative paraffin sections of rat lung tissue stained with hematoxylin and eosin as function of time for sham-operated group (sham), control group (saline $+I+R$ ), and groups with surfactant administered before (surfactant $+I+R$ ) and after $(I+$ surfactant $+R$ ) ischemia. Original magnification $\times 200$.

to those in the sham-operated group (Figure 5). Surfactant reduced cleaved caspase- 3 expression at 120 minutes of reperfusion relative to control ( $P<.001 \mathrm{SIR}$ and ISR). Also at 30 and 240 minutes of reperfusion, caspase-3 levels were diminished in both surfactant groups (SIR and ISR) relative to both the control and sham-operated groups $(P<.05$; Figure 5$)$.

\section{DISCUSSION}

In this study, we determined the effects of exogenous surfactant on inflammation and apoptosis in an animal model of LIRI. Although many studies have described the protective effect of exogenous surfactant on lung function and outcome in experimental models of acute lung injury, ${ }^{2,3,6}$ little is known about the immunomodulatory effects of surfactant in vivo. The effects of surfactant seem to depend on the experimental model, dosage, and time of administration. ${ }^{1,4,5}$ To address the time of administration, we treated rats either before ischemia or just after ischemia. We show here that surfactant increases IL-6 and IL-10 in the lung at 240 minutes after ischemia. IL- 6 is a pleiotropic cytokine that can either induce proinflammatory mediator expression or exert cytoprotective effects, depending on the in vivo environmental circumstances. ${ }^{17}$ IL-6 is transcriptionally regulated by neutral factor $\kappa \mathrm{B}$, but at high levels IL- 6 protein in turn may limit neutral factor $\kappa \mathrm{B}$ activity in a negative feedback loop, thereby dampening the inflammatory response. We therefore suggest that the increased IL-6 response seen after surfactant treatment may be anti-inflammatory. This hypothesis is supported by data of Farivar and colleagues, ${ }^{18}$ who showed that recombinant IL-6 improved LIRI. Moreover, in a rodent model of hemorrhagic shock, exogenous IL-6 decreased lung and liver tissue injury. ${ }^{19}$

In addition to increased levels of IL-6, we also observed augmented IL-10 expression after surfactant treatment. IL10 has a strong anti-inflammatory effect by limiting the secretion of proinflammatory cytokines by macrophages and $\mathrm{T}$ cells. Several studies have shown that exogenous IL-10 attenuates experimental reperfusion injury of the intestine, liver, lung, and hindlimb. ${ }^{20-23}$ In this study, IL-10 decreased dramatically with time in the control animals, whereas surfactant treatment normalized or even increased IL-10 expression, suggesting that surfactant induces a strong anti-inflammatory environment.

We also looked at the expression of iNOS. Baron and associates ${ }^{24}$ demonstrated that expression of iNOS in lung epithelial cells is critical for the development of lung injury and furthermore mediates surfactant dysfunction. Some investigators have suggested that increased lung apoptosis could be one of the mechanisms through which iNOS causes lung injury. ${ }^{25}$ We report that iNOS was specifically upregulated in the control group, whereas it was not upregulated in the sham-operated group, which supports the notion that iNOS is an important mediator during lung injury. In line with this finding, we observed increased cleaved caspase-3 expression in the control group. Interestingly, 2 and 4 hours after ischemia iNOS was strongly upregulated by surfactant either before (SIR) or after (ISR) ischemia. The enhanced expression of iNOS after surfactant treatment was not associated with enhanced apoptosis, because at all time points both surfactant treatment schedules significantly decreased 


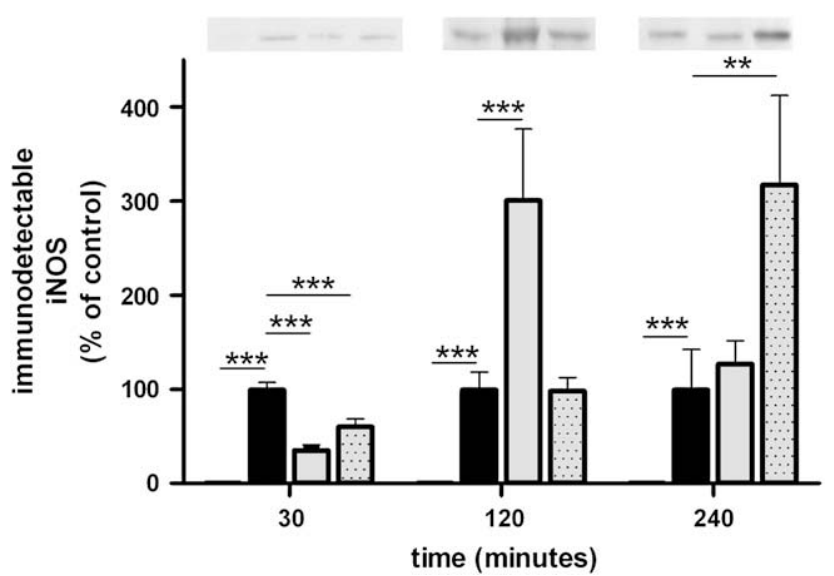

FIGURE 2. Inducible nitric oxide ( $i N O S$ ) concentrations as function of time. Inducible nitric oxide expression levels were assessed in total cell ysates resolved by $10 \%$ sodium dodecyl sulfate polyacrylamide gel electrophoresis and analyzed by Western blot. Data (mean \pm SEM) were normalized to $\beta$-actin expression and depicted as percentage of control values (black bars). Insets are representative Western blots depicting immunodetectable inducible nitric oxide. $* * P<.01 ; * * * P<.001 ; \mathrm{N}=7$ per group. $\square$ sham; $\square$ saline $+\mathrm{I}+\mathrm{R} ; \square$ surfactant $+\mathrm{I}+\mathrm{R}$; $\equiv \mathrm{I}+$ surfactant $+\mathrm{R}$.

apoptosis in the lung. Interestingly, there are some indications that iNOS expression may be involved in antiapoptotic pathways, depending on cell type, stimulus, and duration (as reviewed by $\mathrm{Mehta}^{25}$ ). In our study, the early expression of iNOS, 30 minutes after ischemia, was significantly downregulated by surfactant treatment. We therefore speculate that early expression of iNOS triggers the proinflammatory response, whereas longer periods of iNOS expression trigger antiapoptotic pathways. This speculation is supported by the data of Mikawa and coworkers, ${ }^{26}$ who showed that selective inhibition of iNOS with ONO-1714 improved lung oxygenation, pulmonary edema, and leukocyte sequestration when administered before or within 2 hours after induction



FIGURE 3. Interleukin 6 (IL-6) concentrations as function of time. Interleukin 6 levels were determined by standard enzyme-linked immunosorbent assay. $* P<.05 ; * * P<.01 ; \mathrm{N}=7$ per group. $\square$ sham; $\square$ saline $+\mathrm{I}+\mathrm{R}$; $\square$ surfactant $+\mathrm{I}+\mathrm{R}$; 册 I+surfactant $+\mathrm{R}$.

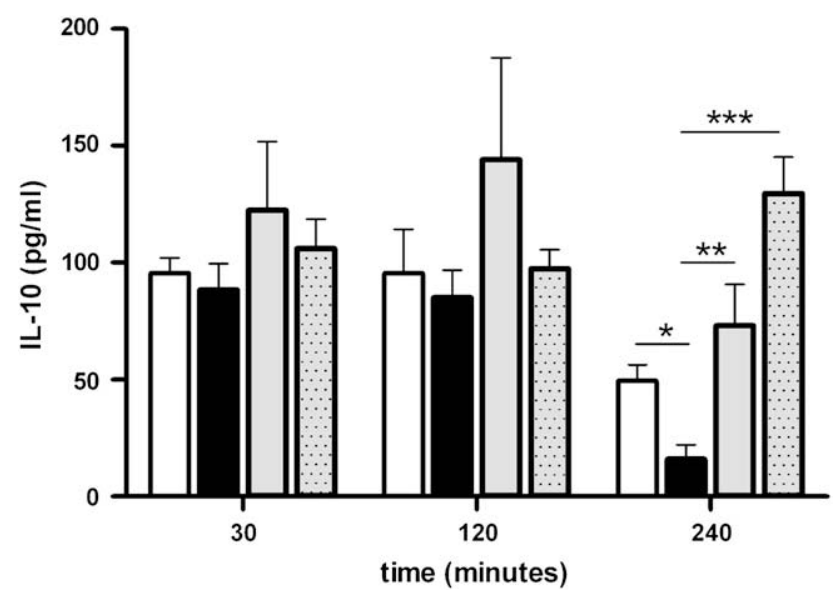

FIGURE 4. Interleukin $10(I L-10)$ concentrations as function of time. Interleukin 10 levels were determined by standard enzyme-linked immunosorbent assay. $* P<.05 ; * * P<.01 ; * * * P<.001 ; \mathrm{N}=7$ per group. $\square$ sham; m saline $+\mathrm{I}+\mathrm{R}$; $\square$ surfactant $+\mathrm{I}+\mathrm{R}$; 曲 $\mathrm{I}+$ surfactant $+\mathrm{R}$.

of acute lung injury but not if administered 3 or 4 hours after induction.

Interestingly, comparison of the effects of surfactant administered before and after ischemia on the parameters measured showed a time-dependent relationship. The delayed response in the ISR group is explained by the time sequence of the experimental setting. It is known that the reperfusion period plays a significantly more important role in causing injury than does the ischemic period. ${ }^{27}$ This might explain the equivalent responses, but with different time kinetics,

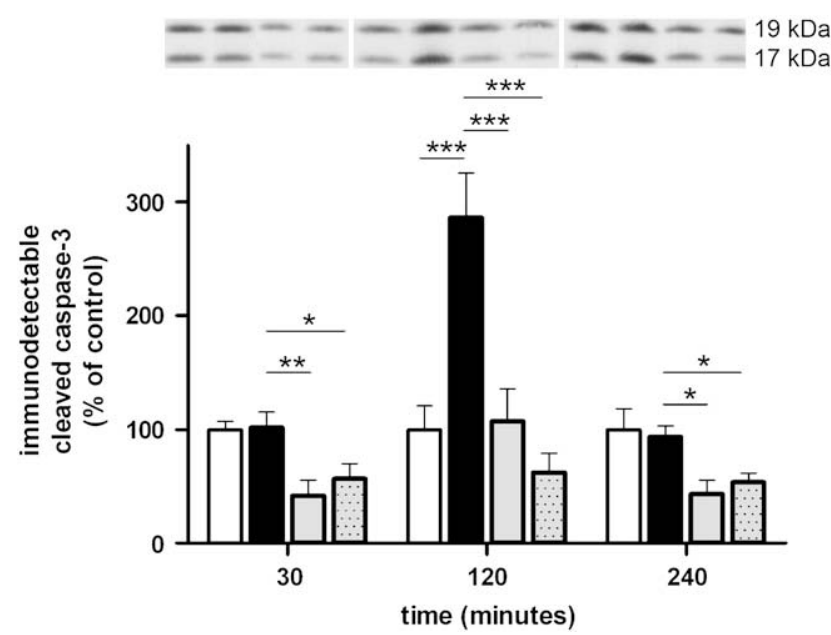

FIGURE 5. Effect of lung ischemia-reperfusion injury on cleaved caspase- 3 expression as function of time. Cleaved caspase- 3 expression levels were assessed in total cell lysates resolved by $15 \%$ sodium dodecyl sulfate polyacrylamide gel electrophoresis and analyzed by Western blot. Data (mean $\pm \mathrm{SEM}$ ) were normalized to $\beta$-actin expression and depicted as percentage of values in sham-operated group. Insets are representative Western blots depicting immunodetectable cleaved caspase- $3 . * P<.05 ; * * P<.01$; $* * * P<.001 ; \mathrm{N}=7$ per group. $\square$ sham; $\square$ saline $+\mathrm{I}+\mathrm{R} ; \square$ surfactant $+\mathrm{I}$ $+\mathrm{R} ;$ 曲 $\mathrm{I}+\operatorname{surfactan} \mathrm{t}+\mathrm{R}$. 
that we observed after both surfactant treatment schedules. From a clinical point of view, it may be interesting for future studies to analyze the effect on ischemia-reperfusion injury of surfactant administered after the onset of reperfusion. Interestingly, however, Struber and colleagues ${ }^{28}$ showed that exogenous surfactant instilled in donor lungs before retrieval had protective effects on posttransplant surfactant function and on clinical outcome. ${ }^{28}$

In conclusion, we have shown surfactant to have pleiotropic effects during LIRI. First, surfactant therapy efficiently improves lung architecture. Second, surfactant results in anti-inflammation, accompanied by decreased apoptosis. Finally, surfactant decreases the short-term iNOS expression but increases the long-term iNOS expression. Surfactant replacement therapy thus may provide a good approach to treat LIRI in patients, although more studies are warranted to further delineate the long-term effects of surfactant and the mechanisms involved.

\section{References}

1. van Helden HP, Kuijpers WC, Langerwerf PE, Langen RC, Haagsman HP, Bruijnzeel PL. Efficacy of Curosurf in a rat model of acute respiratory distress syndrome. Eur Respir J. 1998;12:533-9.

2. Chen CM, Fang CL, Chang CH. Surfactant and corticosteroid effects on lung function in a rat model of acute lung injury. Crit Care Med. 2001;29:2169-75.

3. Verbrugge SJ, Vazquez de Anda G, Gommers D, Neggers SJ, Sorm V, Böhm SH, et al. Exogenous surfactant preserves lung function and reduces alveolar Evans blue dye influx in a rat model of ventilation-induced lung injury. Anesthesiology. 1998;89:467-74.

4. Hausen B, Rohde R, Hewitt CW, Schroeder F, Beuke M, Ramsamooj R, et al. Exogenous surfactant treatment before and after sixteen hours of ischemia in experimental lung transplantation. J Thorac Cardiovasc Surg. 1997;113:1050-8.

5. Novick RJ, MacDonald J, Veldhuizen RA, Wan F, Duplan J, Denning. L et al. Evaluation of surfactant treatment strategies after prolonged graft storage in lung transplantation. Am J Respir Crit Care Med. 1996;154:98-104.

6. Erasmus ME, Petersen AH, Hofstede G, Haagsman HP, Bambang OS, Prop J. Surfactant treatment before reperfusion improves the immediate function of lung transplants in rats. Am J Respir Crit Care Med. 1996;153:665-70.

7. Wright JR. Immunoregulatory functions of surfactant proteins. Nat Rev Immunol. 2005;5:58-68.

8. Antal JM, Divis LT, Erzurum SC, Wiedemann HP, Thomassen MJ. Surfactant suppresses NF- $\kappa$ B activation in human monocytic cells. Am J Respir Cell Mol Biol. 1996;14:374-9.

9. Thomassen MJ, Antal JM, Connors MJ, Meeker DP, Wiedemann HP. Characterization of exosurf (surfactant)-mediated suppression of stimulated human alveolar macrophage cytokine responses. Am J Respir Cell Mol Biol. 1994;10:399-404.

10. Baur FM, Brenner B, Goetze-Speer B, Neu S, Speer CP. Natural porcine surfactant (Curosurf) down-regulates mRNA of tumor necrosis factor-alpha (TNF- $\alpha$ ) and TNF- $\alpha$ type II receptor in lipopolysaccharide-stimulated monocytes. Pediatr Res. 1998;44:32-6.

11. Vreugdenhil HA, Lachmann B, Haitsma JJ, Zijlstra J, Heijnen CJ, Jansen NJ, et al. Exogenous surfactant restores lung function but not peripheral immunosuppression in ventilated surfactant-deficient rats. Exp Lung Res. 2006;32:1-14.

12. Krause MF, Wiemann T, Reisner A, Orlowska-Volk M, Köhler H, Ankermann T. Surfactant reduces extravascular lung water and invasion of polymorphonuclear leukocytes into the lung in a piglet model of airway lavage. Pulm Pharmacol Ther. 2005; 18:129-39.

13. Kinniry P, Pick J, Stephens S, Jain D, Solomides CC, Niven R, et al. KL4-surfactant prevents hyperoxic and LPS-induced lung injury in mice. Pediatr Pulmonol. 2006;41:916-28.

14. van Kaam AH, Lutter R, Lachmann RA, Haitsma JJ, Herting E, Snoek M, et al. Effect of ventilation strategy and surfactant on inflammation in experimental pneumonia. Eur Respir J. 2005;26:112-7.

15. Stamme C, Brasch F, von Bethmann A, Uhlig S. Effect of surfactant on ventilation-induced mediator release in isolated perfused mouse lungs. Pulm Pharmacol Ther. 2002; 15:455-61.

16. Rasaiah VP, Malloy JL, Lewis JF, Veldhuizen RA. Early surfactant administration protects against lung dysfunction in a mouse model of ARDS. Am J Physiol Lung Cell Mol Physiol. 2003;284:L783-90.

17. Heinrich PC, Behrmann I, Haan S, Hermanns HM, Muller-Newen G, Schaper F. Principles of interleukin (IL)-6-type cytokine signalling and its regulation. Biochem J. 2003;374:1-20.

18. Farivar AS, Merry HE, Fica-Delgado MJ, McCourtie AS, MackinnonPatterson BC, Mulligan MS. Interleukin-6 regulation of direct lung ischemia reperfusion injury. Ann Thorac Surg. 2006;82:472-8.

19. Meng ZH, Dyer K, Billiar TR, Tweardy DJ. Distinct effects of systemic infusion of G-CSF vs. IL-6 on lung and liver inflammation and injury in hemorrhagic shock. Shock. 2000;14:41-8.

20. Dinant S, Vetelainen RL, Florquin S, van Vliet AK, van Gulik TM. IL-10 attenuates hepatic I/R injury and promotes hepatocyte proliferation. J Surg Res. 2007; 141:176-82.

21. Hess PJ, Seeger JM, Huber TS, Welborn MB, Martin TD, Harward TR, et al. Exogenously administered interleukin-10 decreases pulmonary neutrophil infiltration in a tumor necrosis factor-dependent murine model of acute visceral ischemia. J Vasc Surg. 1997;26:113-8.

22. Engles RE, Huber TS, Zander DS, Hess PJ, Welborn MB, Moldawer LL, et al. Exogenous human recombinant interleukin-10 attenuates hindlimb ischemiareperfusion injury. J Surg Res. 1997;69:425-8.

23. Eppinger MJ, Ward PA, Bolling SF, Deeb GM. Regulatory effects of interleukin-10 on lung ischemia-reperfusion injury. J Thorac Cardiovasc Surg. 1996;112:1301-5.

24. Baron RM, Carvajal IM, Fredenburgh LE, Liu X, Porrata Y, Cullivan ML, et al. Nitric oxide synthase-2 down-regulates surfactant protein-B expression and enhances endotoxin-induced lung injury in mice. FASEB J. 2004;18:1276-8.

25. Mehta S. The effects of nitric oxide in acute lung injury. Vascul Pharmacol. 2005; 43:390-403.

26. Mikawa K, Nishina K, Takao Y, ONO-1714 Obara H. a nitric oxide synthase inhibitor, attenuates endotoxin-induced acute lung injury in rabbits. Anesth Analg. 2003;97:1751-5.

27. Ng CS, Wan S, Arifi AA, Yim AP. Inflammatory response to pulmonary ischemia-reperfusion injury. Surg Today. 2006;36:205-14.

28. Struber M, Fischer S, Niedermeyer J, Warnecke G, Gohrbandt B, Gorler A, et al. Effects of exogenous surfactant instillation in clinical lung transplantation: a prospective, randomized trial. J Thorac Cardiovasc Surg. 2007;133:1620-5. 